\title{
Chloroquine inhibits cell growth in human A549 lung cancer cells by blocking autophagy and inducing mitochondrial-mediated apoptosis
}

\author{
LIKUN LIU ${ }^{1}$, CUICUI HAN ${ }^{2}$, HAITAO YU ${ }^{3}$, WENBIN ZHU ${ }^{1}$, HONGXIA CUI ${ }^{4}$, \\ LIHONG ZHENG ${ }^{3}$, CHUNJING ZHANG ${ }^{5}$ and LILING YUE ${ }^{1}$ \\ ${ }^{1}$ Research Institute of Medicine and Pharmacy; Departments of ${ }^{2}$ Pharmacology, ${ }^{3}$ Biology Genetics, \\ ${ }^{4}$ Clinical Pharmacology and ${ }^{5}$ Biochemistry, Qiqihar Medical University, Qiqihar, Heilongjiang 161006, P.R. China
}

Received February 4, 2017; Accepted March 28, 2018

DOI: $10.3892 /$ or.2018.6363

\begin{abstract}
Chloroquine (CQ) has been revealed to exhibit antitumor activity in several human tumors including lung cancer as mono- or add-on therapy. The antitumor effect of CQ appears to depend on the tumor type, stage and genetic context. Few studies have focused on the mechanism concerning the antitumor effect of CQ monotherapy and the cause and effect relationship among autophagy, apoptosis and CQ in human lung A549 cells. Therefore, the present study aimed to identify the antitumor effect of CQ monotherapy and analyze the possible mechanism. In the present study, we demonstrated that CQ suppressed human A549 cell growth in a dose- and time-dependent manner. CQ-mediated growth inhibition in A549 cells was characterized by the targeting of the PI3K/AKT pathway, thus, inducing mitochondria-mediated apoptosis at relatively higher concentrations by downregulating Bcl-2 expression, increasing the expression level of Bax, decreasing mitochondrial membrane potential, releasing cytochrome $c$ from the mitochondria into the cytosol, activating caspase- 3 and cleaving PARP. Collectively, these findings may offer a new rationale for using CQ as a lung cancer therapy drug in clinical practice.
\end{abstract}

\section{Introduction}

Chloroquine (CQ), a 4-aminoquinoline compound, has long been prescribed for the treatment of malaria (1), autoimmune disorders (2) and viral infections (3). It functions primarily by inhibiting lysosomal proteases and blocking autophagosome-lysosome fusion (4). In the range of neutral

Correspondence to: Professor Liling Yue, Research Institute of Medicine and Pharmacy, Qiqihar Medical University, 333 Bukui Street, Jianhua, Qiqihar, Heilongjiang 161006, P.R. China E-mail: yuel11025@126.com

Key words: chloroquine, human A549 cells, cell proliferation, apoptosis, autophagy
$\mathrm{pH}, \mathrm{CQ}$ can freely diffuse across the plasma membrane but can also get protonated and trapped in acidic vesicles such as lysosomes. The accumulation of protonated CQ results in less acidic conditions in lysosomes, further preventing autophagosome-lysosome fusion and autophagosome degradation. Nowadays, CQ is receiving more attention from all over the world mostly due to its potent antitumor activity as mono- or add-on therapy. It has been reported that CQ can increase sensitization of radiation (5-7) and chemotherapeutic agents including ABT-737, 5-fluorouracil and PI103 (8-10), and then induce cell death in a subset of cancer cell lines. Recent evidence suggests that CQ alone effectively suppressed the growth of pancreas, leukemia, lung, colon and liver cancer cells (11-15), and promoted apoptosis by increasing the expression of pro-apoptotic protein Bim both in HepG2 and Huh7 cells (15), activating the p53 pathway in glioma cells (16) and stabilizing the $\mathrm{BH} 3$-only protein PUMA in melanoma cells (17). However, the mechanism underlying the antitumor effect of CQ monotherapy in human A549 cells has not been clearly investigated yet.

Autophagy is a self-defense event in all eukaryotic cells triggered by internal needs or extracellular stressors. It mainly serves as a mechanism for cell survival. Normally, autophagy functions in the maintenance of cellular homeostasis by delivering impaired organelles or unwanted cellular components to the lysosomes for degradation and recycling (18). In recent years, there has been an increasing amount of research on the relationship between autophagy and cancer. The role of autophagy in cancer is highly complex and still paradoxical. It appears to have the double-edged sword effect: Under stress circumstances, autophagy can protect cancer cells against apoptosis and/or other forms of cell death by providing energy and essential macromolecules, whereas excessive autophagy may cause irreversible self-destruction of cancer cells and further induce autophagic cell death (19). Notably, several proteins functioning in the process of apoptosis are also required for autophagy. Autophagy and apoptosis can be triggered by the same stimuli. They are closely interconnected at some special points of crosstalk in different types of cancer (20). Thus, thoroughly analyzing the mechanism of crosstalk between autophagy and apoptosis is essential for 
successful anticancer treatments as a potential therapeutic strategy.

Although CQ displays antitumor activity in human A549 cells, there are very few studies that have described the underlying mechanism of the antitumor effect of CQ as a monotherapy and the relationship among autophagy, apoptosis and CQ. Therefore, the present study was designed to investigate the antitumor effect of CQ in human A549 cells, further analyze the possible molecular mechanism of $\mathrm{CQ}$ monotherapy in the regulation of autophagy or apoptosis, as well as the association between autophagy and apoptosis when the A549 cells were exposed to CQ. Thus, it may offer a solid experimental base for utilizing CQ as a monotherapy agent in cancer therapy in the future.

\section{Materials and methods}

Cell line culture and reagents. Human lung A549 cells and human kidney 293T cells were obtained from the Cell Bank of the Chinese Academy of Sciences (Shanghai, China), cultured in Ham's F-12 medium (Sigma-Aldrich Chemie GmbH, Munich, Germany) and Dulbecco's modified Eagle's medium (DMEM; HyClone Laboratories, Logan, UT, USA), respectively, supplemented with $10 \%$ fetal bovine serum (FBS; HyClone, Laboratories), $100 \mathrm{U} / \mathrm{ml}$ penicillin and $100 \mu \mathrm{g} / \mathrm{ml}$ streptomycin (Gibco, Grand Island, NY, USA) at $37^{\circ} \mathrm{C}$ and $5 \% \mathrm{CO}_{2}$ in a humidified atmosphere. $\mathrm{CQ}$ was purchased from Sigma-Aldrich (Merck KGaA, Darmstadt, Germany) and dissolved in deionized distilled water (DDW) until the experiments.

Cell viability assay. The antiproliferative effect of CQ in A549 cells was assessed by MTT assay. Briefly, A549 cells were seeded into 96 -well plates $\left(5 \times 10^{3}\right.$ cells/well) and treated with CQ at different concentrations $(1.25,2.5,5,10,20,40$, 80 and $160 \mu \mathrm{M})$ for 24 or $48 \mathrm{~h}$, respectively. A total of $20 \mu \mathrm{l}$ methylthiazol tetrazolium (MTT) solution (final concentration of $0.5 \mathrm{mg} / \mathrm{ml}$ ) was added and incubated at $37^{\circ} \mathrm{C}$ for another $4 \mathrm{~h}$. Subsequently, $150 \mu \mathrm{l}$ dimethyl sulfoxide (DMSO) was added to dissolve the blue formazan product. The absorbance values were measuring at $570 \mathrm{~nm}$ with an enzyme-labeling instrument (Safire2; Tecan Group, Ltd., Männedorf, Switzerland).

Immunofluorescence of LC3-II. The amount of LC3-II, an indicator of autophagosomes, was detected following a standard procedure of immunofluorescence. Firstly, A549 cells after being treated with or without CQ for $24 \mathrm{~h}$ were grown on sterilized glass coverslips to $90-95 \%$ confluence and fixed with a $4 \%(\mathrm{w} / \mathrm{v})$ paraformaldehyde solution. After being washed 3 times with phosphate-buffered saline (PBS; Sigma Chemical Co., St. Louis, MO, USA), the cells were blocked with $0.1 \%$ Triton X-100 containing $1 \%$ bovine serum albumin (BSA) in PBS for $1 \mathrm{~h}$, and then incubated with LC3B primary antibody (1:200; cat. no. 2775; Cell Signaling Technology, Inc., Danvers, MA, USA) at $4^{\circ} \mathrm{C}$ overnight and subsequently with the corresponding FITC-linked anti-rabbit secondary antibody (1:160; cat. no. F9887; Sigma-Aldrich) for $40 \mathrm{~min}$ at room temperature. Finally, the cells were rinsed with PBS and examined using a fluorescence microscope (Zeiss Axio ObserveRA1; Carl Zeiss GmbH, Jena, German) in 3 random fields.

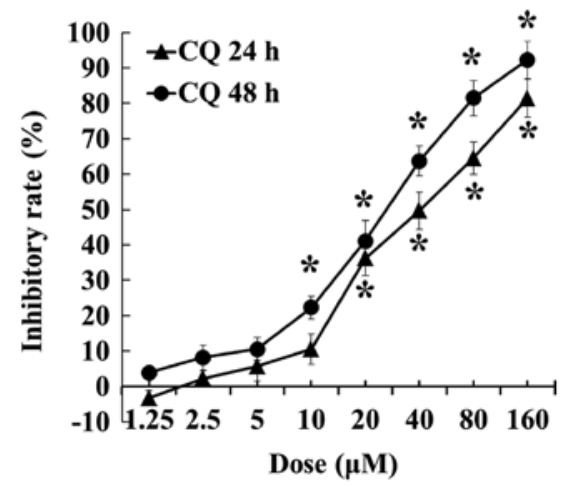

Figure 1. CQ inhibits the proliferation of human A549 cells. The cells were seeded into 96 -well plates $\left(5 \times 10^{3}\right.$ cells/well), and treated with CQ at different concentrations (1.25-160 $\mu \mathrm{M})$ for 24 or $48 \mathrm{~h}$, respectively. Cell viability was assessed by MTT assay. The percentage of proliferation inhibition was calculated by the following formula: inhibition rate $(\%)=(1-$ absorbance of the experimental group/absorbance of the control group) $x 100 \%$. The data are presented as the means $\pm \mathrm{SD}$ of 3 independent experiments. ${ }^{*} \mathrm{P}<0.05$ compared with the control group.

Transmission electron microscopy (TEM) observation. The ultrastructure of human A549 cells were observed using a transmission electron microscope (HT7700; Hitachi, Tokyo, Japan). After CQ administration, the cells were harvested and fixed in $2.5 \%$ glutaraldehyde at $4^{\circ} \mathrm{C}$ overnight. After being washed 3 times with a sucrose solution, the fixed cells were incubated with $1 \%$ osmium tetroxide for $2 \mathrm{~h}$ at room temperature. Subsequently, the cells were dehydrated with gradient ethanol and embedded in Spurr's resin. Ultrathin sections (40-70 nm) were obtained using an ultramicrotome and stained with lead citrate/uranyl acetate and subsequently visualized with a transmission electron microscope at $80 \mathrm{kV}$.

Lentiviral shRNA vector construction and transfection. The interfering sequence targeting the human Beclin-1 gene (GeneBank accession: NM_003766.3) was designed as follows: shRNA, 5'-GCTCAGTATCAGAGAGAATAC-3'. The sequence, 5'-TTCTCCGAACGTGTCACGT-3', sharing no homology with any other human gene was used as a negative control. The annealed, double-stranded DNA was inserted into the lentiviral vector LV2-pGLV-u6-puro. The recombinant plasmids were transformed into DH5 $\alpha$ and the positive colonies selected by PCR were sequenced. 293T cells were co-transfected with $20 \mu \mathrm{g}$ of lentiviral expression plasmid and packaging plasmid (15 $\mu \mathrm{g}$ pHelper 1.0 and $10 \mu \mathrm{g}$ pHelper 2.0) using Lipofectamine 2000 (Invitrogen; Thermo Fisher Scientific, Inc., Waltham, MA, USA). The recombinant lentiviral particles which were obtained $48 \mathrm{~h}$ after transfection were harvested to infect human A549 cells. The interference efficiency was assessed using western blot analysis.

AO/EB dual fluorescence staining. Acridine orange/ethidium bromide $(\mathrm{AO} / \mathrm{EB})$ staining was performed for the observation of morphological changes in cultured cells. Human A549 cells were seeded in a 6 -well plate at a density of $5 \times 10^{5}$ cells $/ \mathrm{ml}$ and incubated overnight before treatment. Then, the cells were exposed to CQ at final concentrations of 0,10, 20, 40 and $80 \mu \mathrm{M}$ for $24 \mathrm{~h}$. Untreated cells were used as the negative 
A

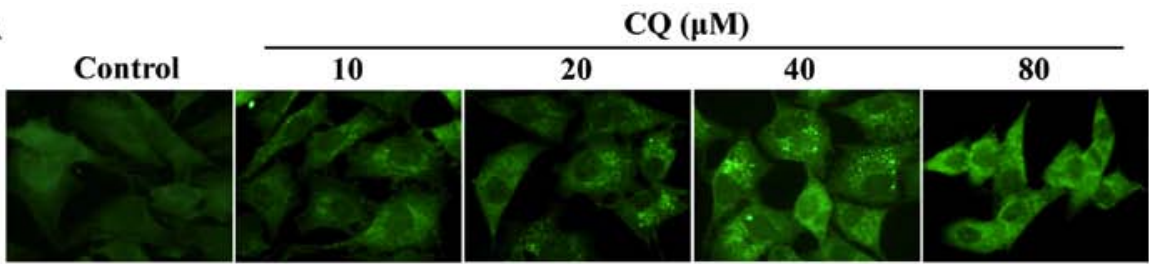

B

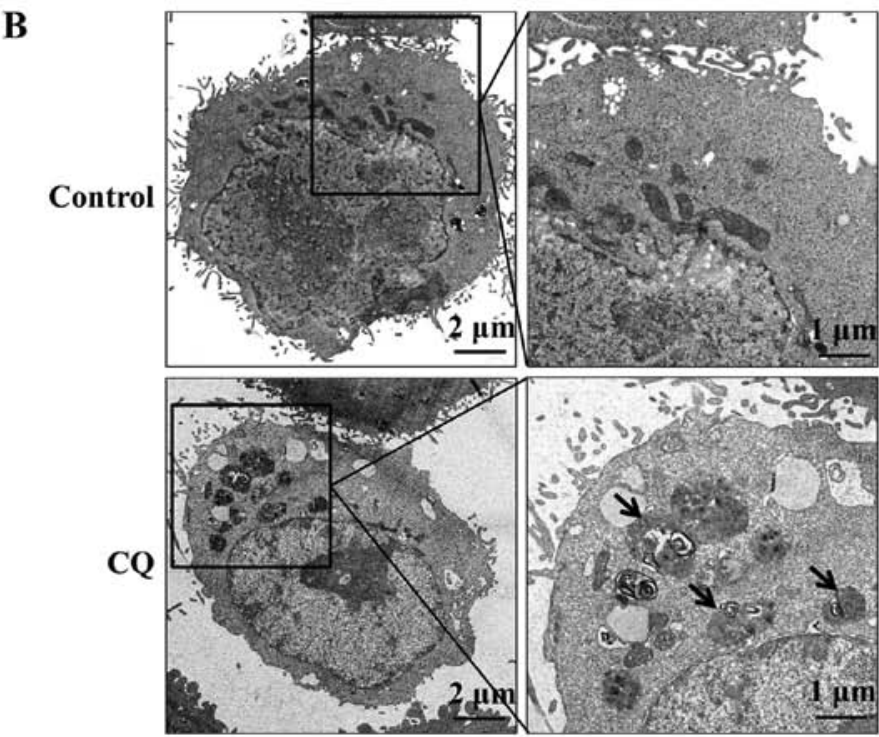

C
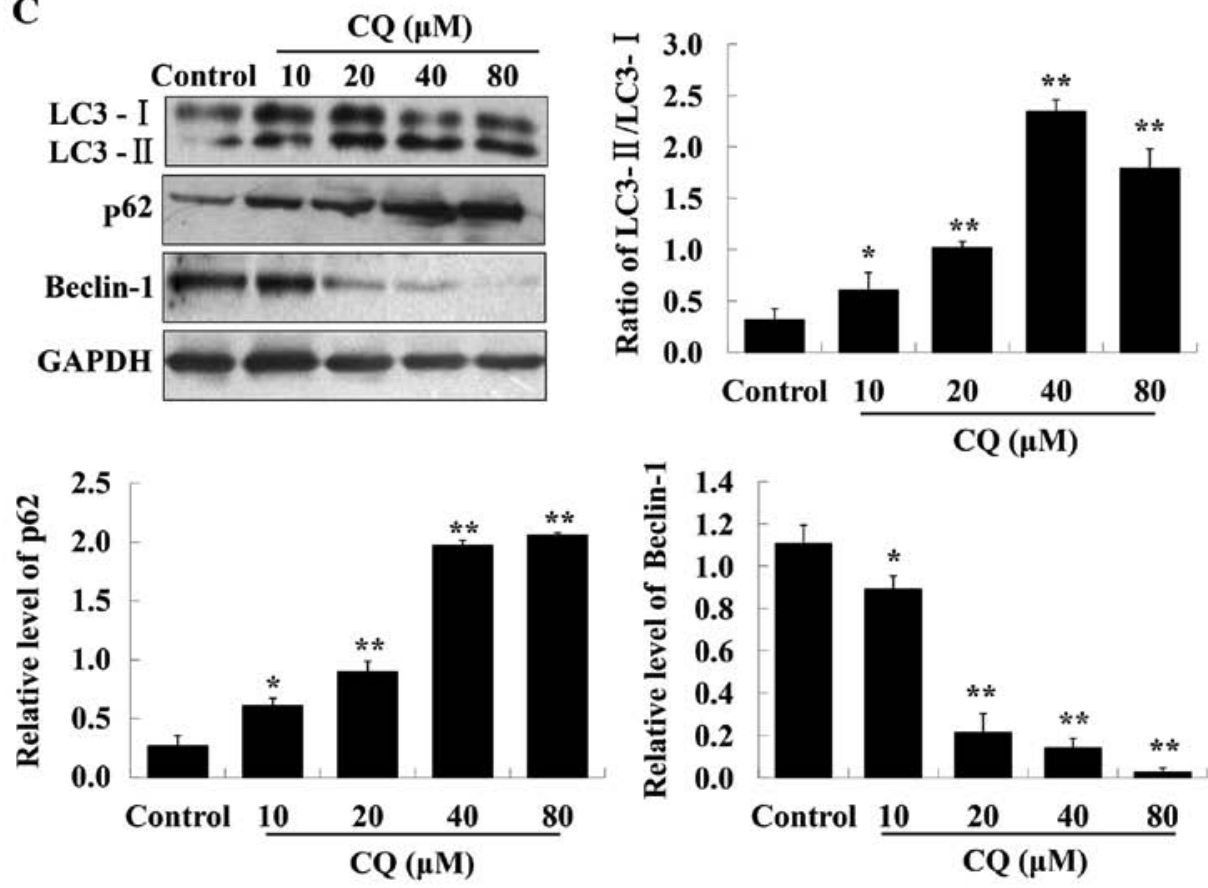

Figure 2. CQ inhibits autophagic flux in human A549 cells. (A) Cells were treated with CQ at different concentrations $(0,10,20,40$ and $80 \mu \mathrm{M})$ for $24 \mathrm{~h}$. LC3 puncta was detected by immunofluorescence. Images were captured using a fluorescence microscope in 3 random fields. (B) Representative transmission electron micrograph of A549 cells treated with CQ. Typical autophagosomes are magnified and indicated with black arrows. (C) Protein expression was examined using western blot analysis for LC3-I, LC3-II, p62 and Beclin-1. GAPDH was used as an internal control. The data are presented as the means \pm SD of 3 independent experiments. ${ }^{*} \mathrm{P}<0.05$ and ${ }^{* *} \mathrm{P}<0.01$ compared with the control group.

control. In subsequent assays, the cells were harvested and stained with AO/EB solution (mixture of AO $100 \mu \mathrm{g} / \mathrm{ml}$ and EB $100 \mu \mathrm{g} / \mathrm{ml}$ prepared in PBS) at room temperature for $15 \mathrm{~min}$. The morphological changes of A549 cells were observed immediately under a fluorescence microscope (Zeiss Axio Observer A1; Carl Zeiss).
Annexin $V$ binding assay. The rate of apoptosis induced by CQ was quantified using the Annexin V-FITC/PI kit (Nanjing KeyGen Biotech, Co., Ltd., Nanjing, China) following the manufacturer's instructions. A549 cells $\left(5 \times 10^{5}\right.$ cells/well) in the exponential phase were treated with different concentrations of CQ $(0,10,20,40$ and $80 \mu \mathrm{M})$ for $24 \mathrm{~h}$. After 
harvesting, the cells were resuspended with Annexin binding buffer and then incubated with $5 \mu 1$ Annexin V-FITC and $5 \mu 1$ of propodium iodine (PI) solutions at room temperature for $15 \mathrm{~min}$ in the dark. The early apoptotic (Annexin V-FITC-positive) and necrotic/late apoptotic cells (Annexin V-FITC-positive, PI-positive) were quantified by BD FACSCalibur Flow Cytometer (BD Biosciences, San Jose, CA, USA).

Mitochondrial membrane potential assay. Mitochondrial membrane potential $(\Delta \Psi m)$ changes after CQ exposure were detected using the JC-1 Mitochondrial Potential Detection kit (Nanjing KeyGen Biotech) and flow cytometry (FCM). After being treated with CQ, A549 cells were trypsinized and washed twice with cold PBS, and then were stained using JC-1 in PBS for $15 \mathrm{~min}$ at room temperature in the dark, followed by FCM analysis.

Western blot analysis. Human A549 cells were treated with $\mathrm{CQ}$ in designated concentrations for $24 \mathrm{~h}$. Total protein was isolated from the control and treated cells using RIPA lysis buffer (Beyotime Institute of Biotechnology, Shanghai, China). The bicinchoninic acid (BCA) protein assay was employed to determine the protein concentration. For western blot analysis, equal amounts of protein were denatured, separated by $10 \%$ sodium dodecyl sulfate-acrylamide gel, and transferred onto nitrocellulose membranes (Pall Life Sciences, Ann Arbor, MI, USA). The non-specific protein bands were blocked in BSA blocking buffer for $1 \mathrm{~h}$ at room temperature. Subsequently, the membranes were incubated with primary antibodies respectively against GAPDH (1:2,500; cat. no. 2118), LC3B (1:1,000; cat. no. 2775), Beclin-1 (1:1,000; cat. no. 3495), p62 (1:1,000; cat. no. 88588), p-AKT $(1: 2,000$; cat. no. 4060), AKT $(1: 1,000$; cat. no. 9272), p-PI3K (1:1,000; cat. no. 4228), PI3K (1:1,000; cat. no. 4292), Bcl-2 (1:1,000; cat. no. 2872), Bax (1:1,000; cat. no. 2774), cytochrome $c(1: 1,000$; cat. no. 11940), c-caspase-3 (1:1,000; cat. no. 9664) and poly(ADP-ribose) polymerase (PARP) (1:1,000; cat. no. 9542) (Cell Signaling Technology, Inc.) at $4^{\circ} \mathrm{C}$ overnight followed by either a goat anti-rabbit (1:2,500; cat. no. 7074; Cell Signaling Technology, Inc.), or goat anti-mouse HRP-conjugated secondary antibody (1:2,500; cat. no. 7076; Cell Signaling Technology, Inc.) for another $2 \mathrm{~h}$ at room temperature. Finally, the reactive bands were visualized using an enhanced chemiluminescent substrate to HRP (Pierce, Woburn, MA, USA). GAPDH was used as the internal control.

Statistical analysis. All experiments were run independently in triplicate. The data were presented as the mean \pm standard deviation (SD). One-way analysis of variance (ANOVA) was employed to determine the differences between the control and treated groups followed by a Student's t-test for multiple comparisons. A $\mathrm{P}<0.05$ indicated a statistically significant result.

\section{Results}

CQ inhibits the viability of A549 cells. The inhibitory effect of CQ on A549 cells was detected by MTT assay. As shown in
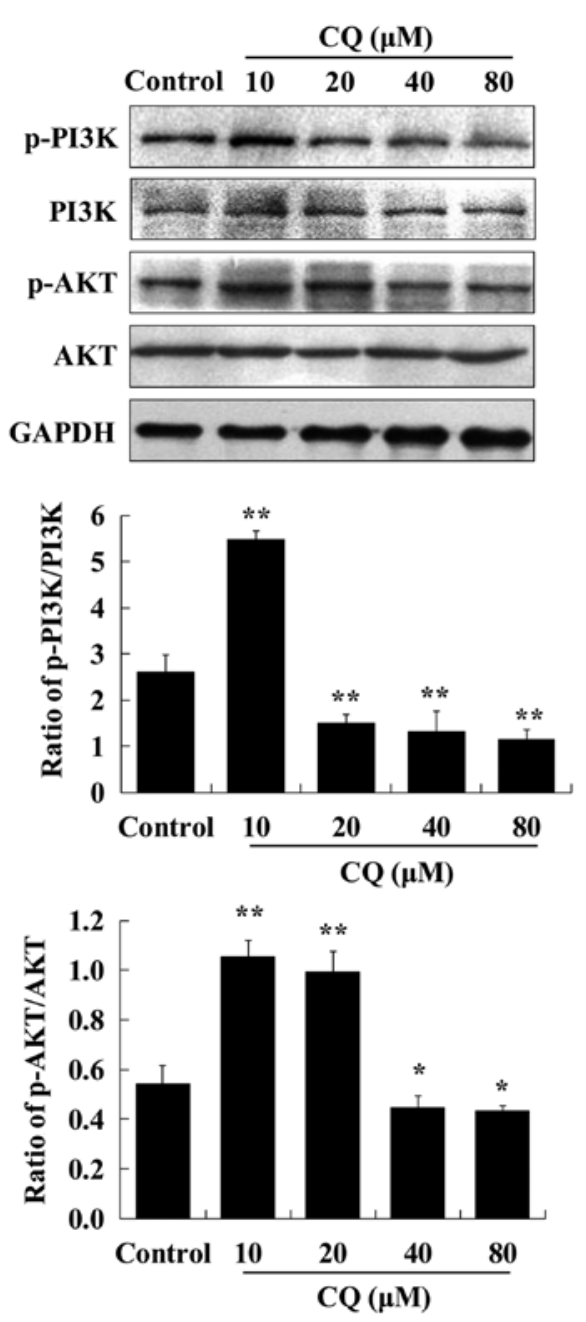

Figure 3. Effect of CQ on the PI3K/AKT pathway in human A549 cells. Cells were treated with CQ at different concentrations $(0,10,20,40$ and $80 \mu \mathrm{M})$ for $24 \mathrm{~h}$. The protein expression was examined using western blot analysis for p-PI3K, PI3K, p-AKT, AKT and GAPDH was used as internal control. The data are presented as the means \pm SD of 3 independent experiments. ${ }^{*} \mathrm{P}<0.05$ and ${ }^{* *} \mathrm{P}<0.01$ compared with the control group.

Fig. 1, CQ inhibited the viability of A549 cells in a dose-and time-dependent manner in CQ concentrations between 2.5 and $160 \mu \mathrm{M}$, and a significant decrease was recorded after the concentration reached $20 \mu \mathrm{M}$ at $24 \mathrm{~h}$ and $10 \mu \mathrm{M}$ at $48 \mathrm{~h}$, respectively $(\mathrm{P}<0.05)$. CQ inhibited cell proliferation to $\sim 80 \%$ at a concentration of $160 \mu \mathrm{M}$ at $24 \mathrm{~h}$ and $92.28 \%$ at a concentration of $160 \mu \mathrm{M}$ at $48 \mathrm{~h}$, respectively.

CQ inhibits autophagic flux in A549 cells. CQ, a typical autophagy inhibitor, contributes to the inhibition of late-stage autophagy by blocking autophagosome-lysosome fusion. We hypothesized that CQ may target autophagy to induce the cell death of A549 cells. LC3 immunofluorescence results indicated that LC3 fluorescence dots were accumulated in A549 cells of the experimental groups, reaching a maximum at a concentration of $40 \mu \mathrm{M}$ of CQ (Fig. 2A). The western blot analysis further confirmed the enhanced expression of LC3-II, whereas that of LC3-I was reduced, resulting in an increased ratio of LC3-II/LC3-I, with the highest ratio reached at $40 \mu \mathrm{M}$ of CQ treatment (Fig. 2C). It is well known that LC3-II specifically associates with autophagosome membranes. A cellular 
A
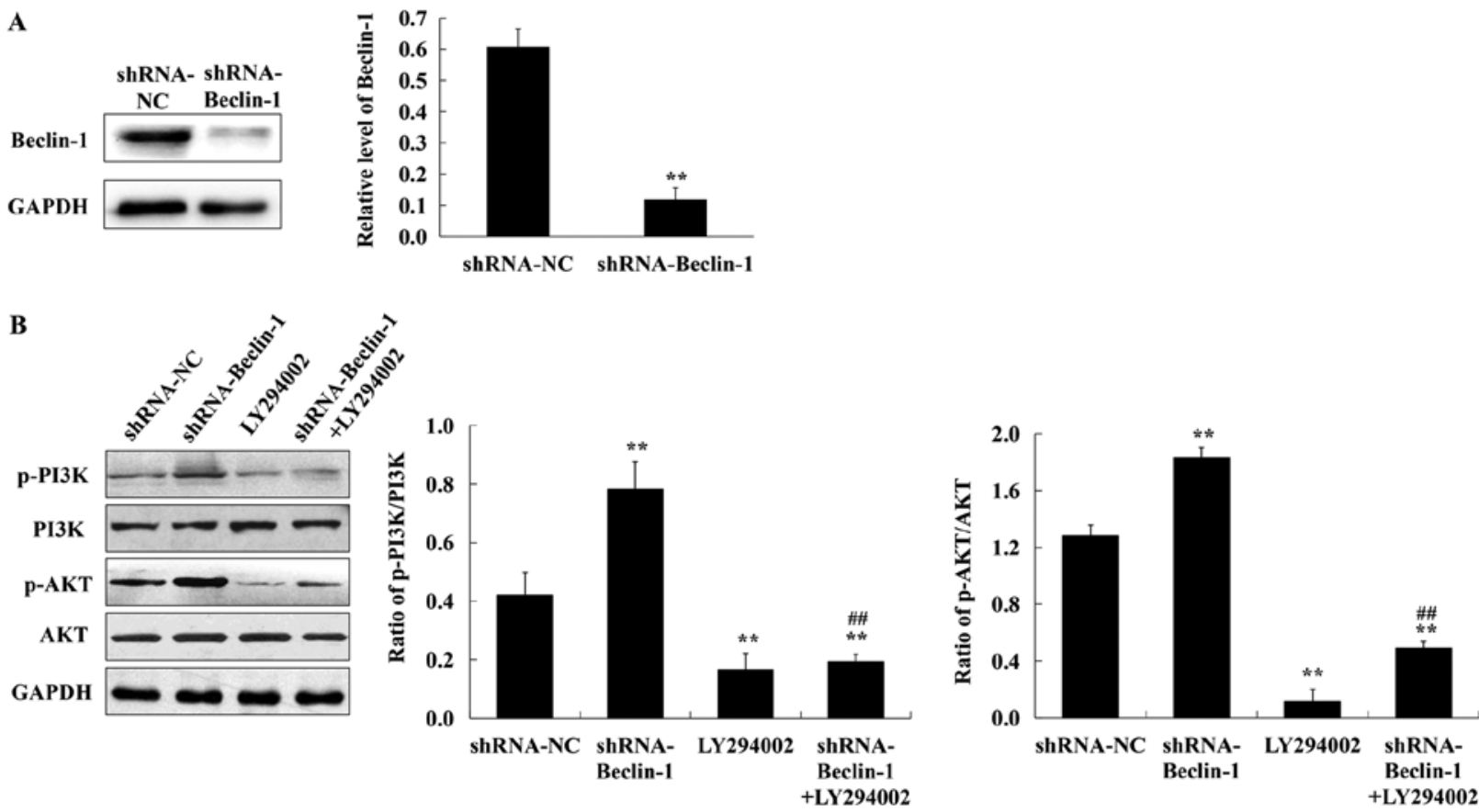

Figure 4. CQ inhibits autophagy by decreasing Beclin-1 expression and activating the PI3K/AKT pathway in human A549 cells. (A) Efficiency of lentiviral shRNA-Beclin-1 interference was detected by western blot analysis. (B) The effect of Beclin-1 knockdown and PI3K inhibitor (LY294002) on the expression of p-PI3K and p-AKT. ${ }^{* *} \mathrm{P}<0.01$ compared with the negative control group; ${ }^{\# \#} \mathrm{P}<0.01$ compared with the shRNA-Beclin-1 group.

level of LC3-II can be used as an autophagosome formation marker (21). TEM-based analysis further confirmed that treatment with CQ markedly increased the number of autophagosomes in A549 cells (Fig. 2B).

The accumulation of autophagosomes may be due to an induction of autophagy or the inhibition of the autophagic flux (22). Beclin-1 has an essential role in autophagy initiation and regulates autophagy positively. p62 is generally used as an autophagic flux marker. The expression of Beclin-1 and p62, were analyzed by western blot analysis. The results demonstrated that exposure of A549 cells to CQ significantly reduced the expression level of Beclin-1. The expression of p62 increased in a dose-dependent manner after treatment with CQ (Fig. 2C). Therefore, these findings revealed that CQ blocks autophagic flux in human A549 cells.

$C Q$ inhibits autophagy by targeting the PI3K/AKT pathway. The process of autophagy is tightly regulated by autophagy-related (ATG) proteins. Beclin-1, the mammalian ortholog of ATG6, governs most autophagic processes and can regulate autophagy positively (23). It was confirmed that the expression levels of Beclin-1 in human A549 cells were downregulated in dose-dependent manner after exposure to $C Q$. The PI3K/AKT signaling pathway is well known as a regulator of various cellular processes, such as cell survival (24). To further explore the mechanisms underlying the autophagy-inhibiting effect of $\mathrm{CQ}$, the expression of critical proteins associated with the PI3K/AKT pathway was examined by western blot analysis in human A549 cells treated with CQ. We observed that the expression of $\mathrm{p}-\mathrm{PI} 3 \mathrm{~K}$ and $\mathrm{p}-\mathrm{AKT}$ was enhanced after $10 \mu \mathrm{M}$ of CQ treatment compared with the control group. Subsequently, a dose-dependent decrease in the expression levels of p-PI3K and p-AKT was observed during exposure to increasing CQ concentrations from 20 to $80 \mu \mathrm{M}$ (Fig. 3). It was speculated that CQ inhibited autophagy at a low concentration $(10 \mu \mathrm{M})$ and induced apoptosis at higher concentrations (20, 40 and $80 \mu \mathrm{M})$.

It was surmised that CQ-inhibited autophagy in A549 cells occurred mainly by reducing Beclin-1 and targeting the PI3K/AKT signaling pathway. To ascertain this conclusion, the lentiviral shRNA-Beclin-1 vector was constructed and the expression of p-PI3K and p-AKT was assessed in A549 cells which were lacking in the Beclin-1 gene in the presence of $50 \mu \mathrm{M}$ LY294002 (a PI3K inhibitor). As revealed in Fig. 4A, the expression of Beclin-1 was significantly reduced after lentivirus shRNA interference. Compared with the negative control, the expression of p-PI3K and p-AKT was increased in the absence of the Beclin-1 gene. LY294002 treatment decreased the expression of p-PI3K and p-AKT in A549 cells transfected with shRNA-Beclin-1 (Fig. 4B). Collectively, these findings confirmed that $\mathrm{CQ}$ inhibited autophagy in human A549 cells by downregulating Beclin-1 and activating the PI3K/AKT signaling pathway.

CQ induces apoptosis in human A549 cells. To determine whether apoptosis induction is involved in the CQ-mediated inhibitory effects on cell growth, rapamycin and HBSS (two autophagy inducers), 3-MA (an autophagy inhibitor), Z-VAD-FMK (a caspase inhibitor) were used in human A549 cells, and their effects on the levels of LC3-II, c-caspase-3 and PARP were assessed by western blot analysis. The results indicated that the level of c-caspase- 3 was markedly enhanced after inhibition of autophagy using 3-MA (Fig. 5). PARP is a specific substrate of caspase-3. If caspase-3 is activated, cleaved PARP can be observed. In the present study, detection of cleaved PARP was possibly due to its faster expression. 

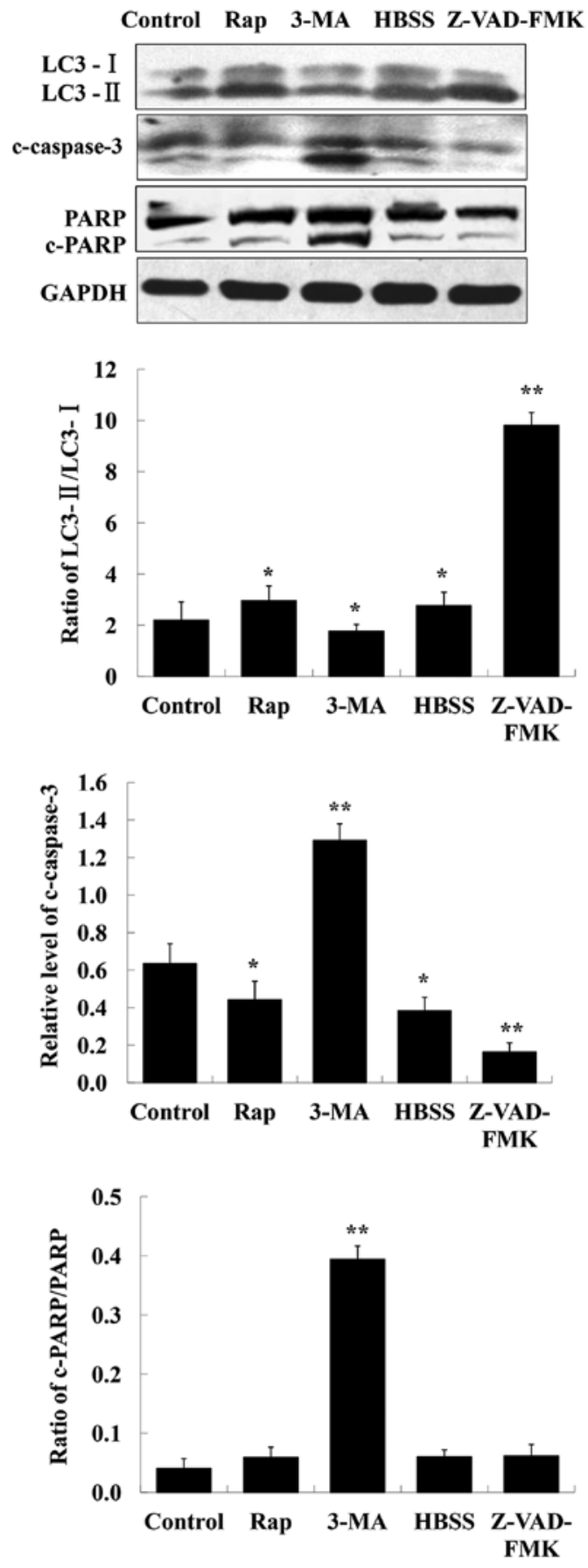

Figure 5. Effect of autophagy inducer, autophagy inhibitor and caspase inhibitor on apoptosis and autophagy in human A549 cells. The protein expression of LC3-I/II, c-caspase-3 and PARP was detected by western blot analysis in cells treated with rapamycin (Rap), HBSS, 3-MA and Z-VAD-FMK, respectively. GAPDH was used as an internal control. The data are presented as the means \pm SD of 3 independent experiments. ${ }^{*} \mathrm{P}<0.05$ and ${ }^{* *} \mathrm{P}<0.01$ compared with the control group.

In addition, it was observed that inhibition of apoptosis by Z-VAD-FMK increased the level of LC3-II whereas that of LC3-I decreased (Fig. 5).

$\mathrm{AO} / \mathrm{EB}$ staining was performed in human A549 cells. As it is well known, AO penetrates the membranes of all cells, fluorescing green when bound to DNA. EB can only enter cells when their membranes are damaged, appearing as orange-red fluorescence when bound to concentrated DNA fragments or apoptotic bodies. Moreover, the fluorescence intensity of EB is stronger than that of AO. This method allows us to distinguish normal cells, early and late apoptotic cells and necrotic cells (25). As demonstrated in Fig. 6A, compared with the negative control group, treatment with CQ in A549 cells induced evident morphological changes associated with apoptosis, such as adherent cell detachement from the culture surface, cell shrinkage and vacuolization gradually as the concentration of CQ increased. The early apoptotic cells were marked by green AO nuclear staining. Late-stage apoptotic cells revealed concentrated and asymmetrical orange fluorescence. The apoptotic cells were increased by CQ in a concentration-dependent manner.

Apoptosis was also detected using Annexin V-FITC and PI dual staining in A549 cells which can quantitatively distinguish normal cells (Annexin $\mathrm{V}-\mathrm{FITC}^{-} / \mathrm{PI}^{-}$), early apoptotic cells (Annexin $\mathrm{V}$-FITC ${ }^{+} / \mathrm{PI}^{-}$), late apoptotic cells (Annexin V-FITC ${ }^{+} / \mathrm{PI}^{+}$)andnecroticcells $\left(\right.$Annexin V-FITC $/ \mathrm{PI}^{+}$). As shown in Fig. 6B, there was a marked increase of cells in early-stage apoptosis (from $0.40 \%$ in the untreated cells to $1.47,3.04$ and $9.64 \%$ respectively, in the 20,40 and $80-\mu \mathrm{M}$ CQ-treated cells for $24 \mathrm{~h}$ ) and late-stage apoptosis (from $3.45 \%$ in the untreated cells to $4.78,12.02$ and $16.80 \%$ respectively, in the 20,40 and 80- $\mu \mathrm{MCQ}$-treated cells for $24 \mathrm{~h}$ ). No obvious cell apoptosis was observed in the $10-\mu \mathrm{M}$ CQ-treated A549 cells. These results confirmed that CQ treatment induced apoptosis at higher concentrations $(20,40$ and $80 \mu \mathrm{M})$ in A549 cells. The proportion of apoptotic cells increased dose-dependently when they were exposed to different concentrations of CQ.

$C Q$ activates mitochondrial-dependent apoptosis in human A549 cells. Apoptosis is generally associated with the activation of caspases but it is also accompanied by a loss of mitochondrial membrane potential and the release of proapoptotic proteins from the intermembrane space of the mitochondria. Mitochondrial membrane potential in A549 cells with or without CQ treatment was detected using JC-1 Mitochondrial Potential Detection kit. As shown in Fig. 7, compared with the control group, CQ treatment caused a drop in mitochondrial membrane potential in a concentration-dependent manner which was observed by an increase in green fluorescent probe JC-1 (from $2.43 \%$ in the untreated cells to $7.10,18.19$ and $28.43 \%$, respectively in the 20,40 and $80-\mu \mathrm{M}$ CQ-treated cells).

It is known that proteins of the Bcl-2 family and caspase family, along with PARP and cytochrome $c$, play vital roles in the mitochondrial apoptotic pathway (26). To further ascertain whether CQ-induced apoptosis was mitochondrial-dependent, we also investigated the expression of apoptosis-related proteins including Bcl-2, Bax, cytochrome $c$, c-caspase-3 and PARP after treatment with various concentrations of CQ. As depicted in Fig. 8, upregulation of Bax, cytochrome $c$, c-caspase-3 and downregulation of Bcl-2 and PARP was induced by $\mathrm{CQ}$ in a dose-dependent manner. The expression of Bcl-2 was downregulated, whereas that of Bax was upregulated, suggesting that $\mathrm{CQ}$ induced an increase in the $\mathrm{Bax} / \mathrm{Bcl}-2$ ratio. We speculated that $\mathrm{CQ}$ treatment reduced the level of Bcl-2, activated the expression of proapoptotic factor Bax, reducing mitochondria membrane potential which led to cytochrome $c$ escape from the mitochondria into the 
A

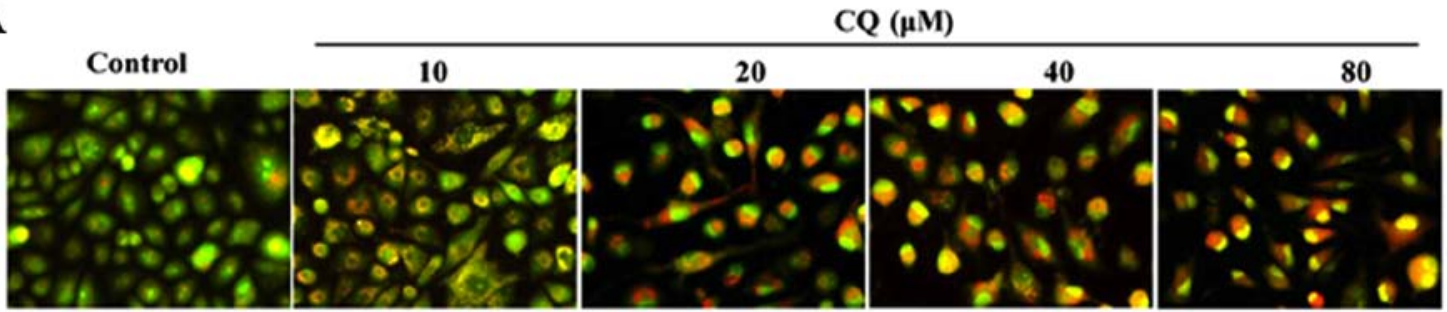

B

$\mathrm{CQ}(\mu \mathrm{M})$

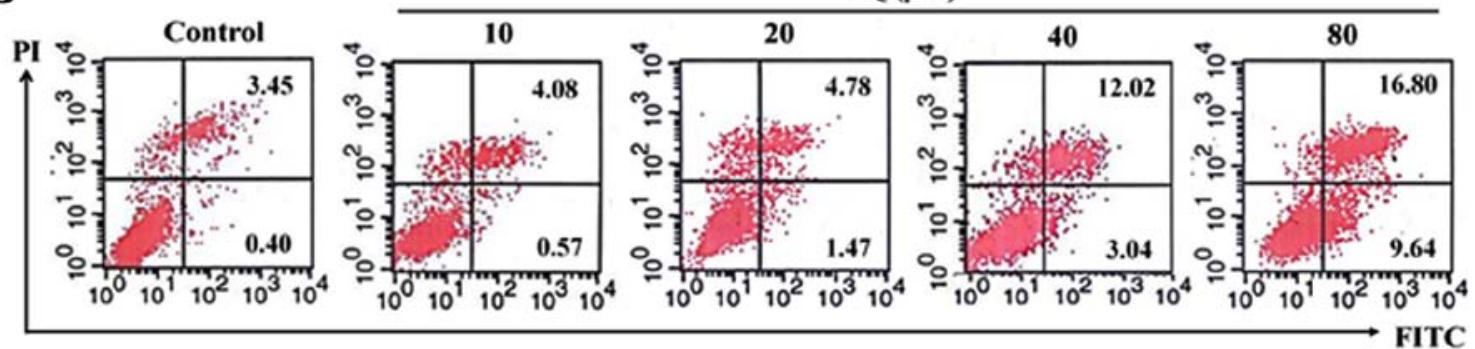

Figure 6. CQ triggers apoptosis of human A549 cells. (A) Cells were treated with CQ at different concentrations ( 0 , $10,20,40$ and $80 \mu \mathrm{M})$ for $24 \mathrm{~h}$, stained with acridine orange/ethidium bromide (AO/EB), and then selected and focused using fluorescence microscopy. Green fluorescence appeared to represent normal and early apoptotic cells, late apoptotic cells presented with red-orange fluorescence in the nucleus. The red-orange fluorescence in the cytoplasm represents autophagic vacuoles. (B) A scattergram of apoptotic A549 cells stained with Annexin V/PI and analyzed by flow cytometry.

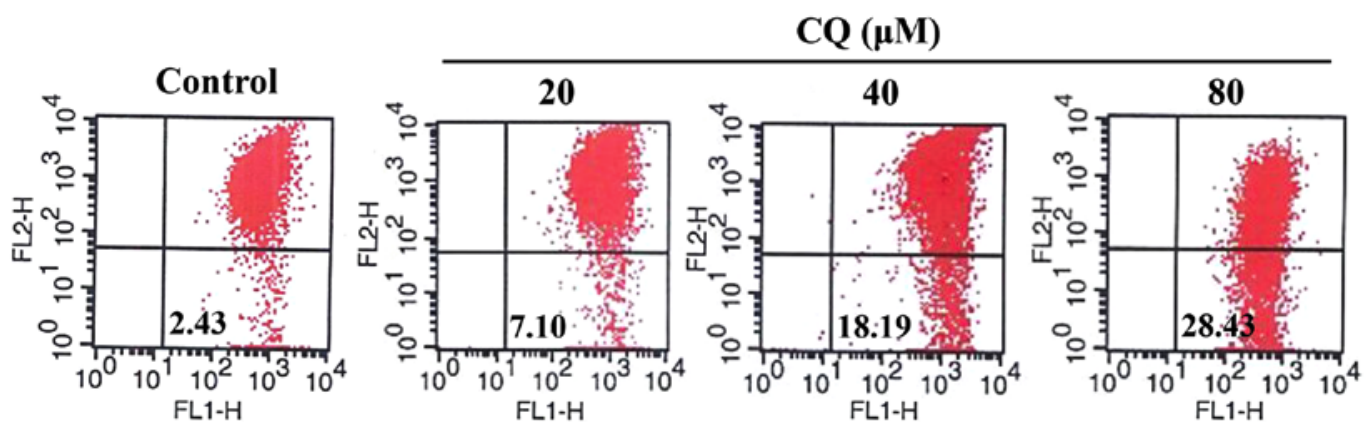

Figure 7. CQ causes a drop in mitochondrial membrane potential $(\Delta \Psi m)$ in A549 cells. A549 cells were treated with CQ at different concentrations $(0,20,40$ and $80 \mu \mathrm{M}$ ) for $24 \mathrm{~h}$. Subsequently, the cell suspension was filtered through a 300-mesh nylon, and then stained with JC-1 followed by FCM analysis.

cytosol, further activating caspase-3 and inducing apoptosis. Collectively, CQ induced cell apoptosis of human A549 cells through the mitochondrial-dependent pathway.

\section{Discussion}

Chloroquine (CQ), is widely used as an anti-malarial and anti-rheumatoid drug (27). Recently, it has been reported that $\mathrm{CQ}$, either alone, or in combination with other agents, displayed antitumor activity, including growth inhibition and/or induction of apoptosis in various types of cancer. The antitumor effect of CQ appears to depend on the tumor type, stage and genetic context (28). Previously, we also revealed that CQ administered as a mono-drug therapy effectively suppressed the growth of S180 sarcoma in vivo (29). Although previous studies have examined the antitumor effect of CQ, only a few studies have focused on the mechanism underlying the effect of CQ and the cause and effect relationship among autophagy, apoptosis and CQ in human A549 cells. Therefore, in the present study, we focused on the antitumor effects of CQ and its possible mechanism. We clearly demonstrated that in vitro, CQ effectively inhibited the proliferation of A549 cells. The inhibitory effect of CQ on proliferation was characterized by the blockage of autophagy through targeting of the PI3K/AKT pathway, coupled with the induction of mitochondrial-mediated apoptosis at relatively higher concentrations.

Conversion of LC3-I into LC3-II is widely used as a marker for autophagosome formation (30). p62 is degraded following an increase in autophagic flux for which it serves as an indicator. The increase of LC3 conversion and p62 abundance suggests the inhibition of autophagic flux. Our results indicated that, marked LC3 conversion and induced p62 accumulation was detected in CQ-treated A549 cells, suggesting that CQ inhibits the autophagic flux. Beclin-1, as a multifaceted protein, is crucial in several cellular processes, such as autophagy, endocytosis, phagocytosis, cytokinesis and pollen germination. Beclin-1 can intervene at every major step in autophagic processes. It functions in the recruitment of ATGs which are essential for autophagosome formation $(31,32)$. The expression level of Beclin-1 can determine the autophagic response. The decreased protein level of Beclin-1 after CQ treatment demonstrated that CQ inhibited autophagy in A549 cells. The results were further confirmed by TEM-based analysis by observing the ultrastructural changes of A549 cells. 

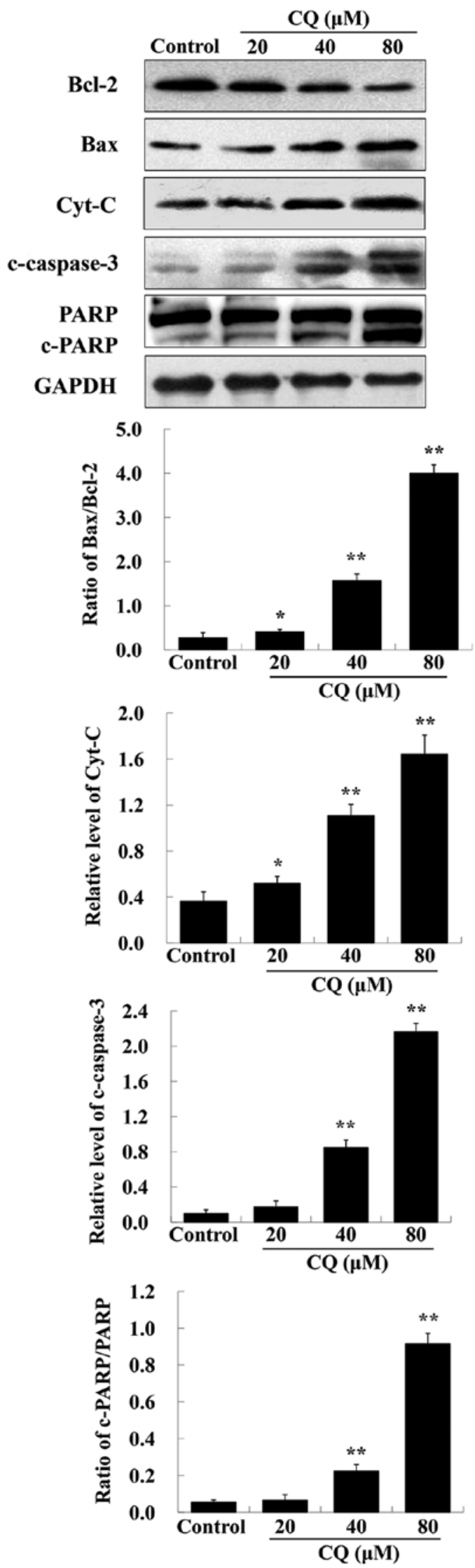

Figure 8. CQ-induced apoptosis of human A549 cells through the activation of the mitochondrial-mediated pathway. Cells were treated with CQ at different concentrations $(0,20,40$ and $80 \mu \mathrm{M})$ for $24 \mathrm{~h}$, and the expression of Bcl-2, Bax, cytochrome $c$ (Cyt-C), c-caspase-3 and PARP was examined, respectively. GAPDH was used as an internal control. The data are presented as the means \pm SD of 3 independent experiments. ${ }^{*} \mathrm{P}<0.05$ and ${ }^{* *} \mathrm{P}<0.01$ compared with the control group.
The PI3K/AKT pathway is an important signaling pathway in the regulation of autophagy (33). Autophagy was impaired by activation of the PI3K/AKT pathway (34). It has been reported that PI3K causes the phosphorylation and activation of AKT. In the present study, it was revealed that CQ at a low concentration induced the phosphorylation of PI3K and AKT, indicating that $\mathrm{CQ}$ at a low concentration mainly inhibits autophagy via the activation of the PI3K/AKT pathway in A549 cells. A dose-dependent decrease in the expression levels of $\mathrm{p}-\mathrm{PI} 3 \mathrm{~K}$ and $\mathrm{p}-\mathrm{AKT}$ during exposure to increasing CQ concentrations from 20 to $80 \mu \mathrm{M}$ revealed that CQ-mediated growth inhibition in A549 cells may be characterized by the inhibition of autophagy and induction of apoptosis.

Previously, research has demonstrated that the biological effect of CQ is concentration- and time-dependent. At low concentrations, CQ inhibits cell proliferation by increasing the volume of lysosomes; at high concentrations, or over longer periods, CQ induces apoptosis and necrosis (12). In this study, CQ effectively inhibited A549 cell proliferation in both a doseand time-dependent manner. Orange vacuoles were observed when cells were exposed to CQ. Accumulation of orange vacuoles was caused by CQ-mediated autophagy inhibition. CQ induced cell apoptosis at relatively higher concentrations. The number of early and late apoptotic cells increased following an increase in CQ concentration. These findings are consistent with results from aforementioned research. Further experiments illustrated that CQ-induced apoptosis was mitochondrial-dependent in A549 cells by downregulating the expression of the anti-apoptotic factor Bcl-2, increasing proapoptotic factor Bax expression, reducing mitochondrial membrane potential, and triggering cytochrome $c$ release into the cytosol, followed by caspase- 3 activation and cleavage of PARP.

Autophagy and apoptosis are both cellular catabolic processes essential for organism homeostasis. Many stimuli elicit autophagy and apoptosis within the same cell. In many cases, autophagy before apoptosis dismantles the cell (19). In special cases, autophagy or autophagy-relevant proteins sensitize cells to apoptosis or necrosis, leading to autophagic cell death. Autophagy and apoptosis may be triggered by common upstream signals. The PI3K-AKT axis exhibits the dual capacity to regulate both autophagy and apoptosis. AKT can phosphorylate Beclin-1 and BAD, further inhibiting their pro-autophagic and pro-apoptotic activity, respectively (35). Beclin-1 and Bcl-2 have also been implicated in bridging autophagy and apoptosis. The PI3Kc3 complex controls autophagy by regulating autophagosome formation. Beclin-1, as a key component of the PI3Kc3 complex, works as the platform for assembly and triggers its activity (36). Notably, Beclin-1 can be cleaved in apoptosis by caspases, such as, caspase-3, caspase-7 and caspase- 8 . The cleavage of Beclin-1 loses its capacity to induce autophagy and generates $\mathrm{N}$ - and $\mathrm{C}$-terminal fragments. The $\mathrm{C}$-terminal fragments are able to sensitize cells to apoptotic signals. Bcl-2 is a direct binding partner of Beclin-1. In cells, Bcl-2 is constitutively bound to Beclin-1, leading to decreased autophagic activity. However, Bcl-2 does not lose its anti-apoptotic potential (37).

In summary, the present study demonstrated that CQ at a low concentration inhibited autophagy by targeting the PI3K/AKT pathway. With increased concentration of CQ, apoptosis was markedly triggered through the activation of the 
mitochondrial pathway and CQ effectively inhibited human A549 cell proliferation in vitro. The present study may provide new theoretical and experimental evidence for a clinical trial on CQ in lung cancer patients.

\section{Acknowledgements}

We thank the Molecular Biology Experiment Center (MBEC) at Qiqihar Medical University for the use of shared facilities.

\section{Funding}

The present study was sponsored by the Youth Special Purpose Foundation (grant no. 1253G066) from the Education Department of Heilongjiang Province, the Qiqihar Municipal Science and Technology Project of China (no. SFGG-201556), the Natural Science Foundation of Heilongjiang Province for Returned Scholars of China (no. LC2011C34) and the Key Program from Qiqihar Medical University of China (no. QY2013ZD-02).

\section{Availability of data and materials}

The datasets used during the present study are available from the corresponding author upon reasonable request.

\section{Authors' contributions}

LY conceived and designed research. LL analyzed data and wrote the paper. $\mathrm{CH}, \mathrm{WZ}, \mathrm{HC}$ and $\mathrm{CZ}$ performed the experiments. LY, HY and LZ reviewed and edited the manuscript. HY and LZ were also involved in the conception of the study. All authors read and approved in ensuring that the accuracy or integrity of any part of the work are appropriately investigated and resolved.

\section{Ethics approval and consent to participate}

Not applicable.

\section{Consent for publication}

Not applicable.

\section{Competing interests}

The authors state that they have no competing interest.

\section{References}

1. Homewood CA, Warhurst DC, Peters W and Baggaley VC: Lysosomes, $\mathrm{pH}$ and the anti-malarial action of chloroquine. Nature 235: 50-52, 1972

2. Rainsford KD, Parke AL, Clifford-Rashotte M and Kean WF: Therapy and pharmacological properties of hydroxychloroquine and chloroquine in treatment of systemic lupus erythematosus, rheumatoid and related diseases. Inflammopharmacology 23 231-269, 2015.

3. Savarino A, Di Trani L. Donatelli I, Cauda R and Cassone A: New insights into the antiviral effects of chloroquine. Lancet Infect Dis 6: 67-69, 2006.
4. Poole B and Ohkuma S: Effect of weak bases on the intralysosomal pH in mouse peritoneal macrophages. J Cell Biol 90: 665-669, 1981.

5. Kim SH, Kim JH and Fried J: Enhancement of the radiation response of cultured tumor cells by chloroquine. Cancer 32 : 536-540, 1973.

6. Djordjevic B, Lange CS, Austin JP and Rotman M: Potentiation of radiation lethality in HeLa cells by combined mild hyperthermia and chloroquine. Radiat Res 130: 267-270, 1992.

7. Djordevic B, Lange CS and Rotman M: Potentiation of radiation lethality in mouse melanoma cells by mild hyperthermia and chloroquine. Melanoma Res 2: 321-326, 1992.

8. Zinn RL, Gardner EE, Dobromilskaya L, Murphy S, Marchionni L, Hann CL and Rudin CM: Combination treatment with ABT-737 and chloroquine in preclinical models of small cell lung cancer. Mol Cancer 12: 16, 2013.

9. Sasaki K, Tsuno NH, Sunami E, Tsurita G, Kawai K, Okaji Y, Nishikawa T, Shuno Y, Hongo K, Hiyoshi M, Kaneko M, et al: Chloroquine potentiates the anti-cancer effect of 5-fluorouracil on colon cancer cells. BMC Cancer 10: 370, 2010.

10. Enzenmüller S, Gonzalez P, Debatin KM and Fulda S: Chloroquine overcomes resistance of lung carcinoma cells to the dual PI3K/mTOR inhibitor PI103 by lysosome-mediated apoptosis. Anticancer Drugs 24: 14-19, 2013.

11. Yang S, Wang X, Contino G, Liesa M, Sahin E, Ying H, Bause A, Li Y, Stommel JM, Dell'antonio G, et al: Pancreatic cancers require autophagy for tumor growth. Genes Dev 25: 717-729, 2011.

12. Fan C, Wang W, Zhao B, Zhang S and Miao J: Chloroquine inhibits cell growth and induces cell death in A549 lung cancer cells. Bioorg Med Chem 14: 3218-3222, 2006.

13. Zheng Y, Zhao Y, Deng X, Yang S, Mao Y, Li Z, Jiang P, Zhao X and Wei Y: Chloroquine inhibits colon cancer cell growth in vitro and tumor growth in vivo via induction of apoptosis. Cancer Invest 27: 286-292, 2009.

14. Jiang PD, Zhao YL, Yang SY, Mao YQ, Zheng YZ, Li ZG and Wei YQ: Effects of chloroquine diphosphate on proliferation and apoptosis of human leukemic K562 cells. Zhongguo Shi Yan Xue Ye Xue Za Zhi 16: 768-771, 2008 (In Chinese).

15. Hu T, Li P, Luo Z, Chen X, Zhang J, Wang C, Chen P and Dong Z: Chloroquine inhibits hepatocellular carcinoma cell growth in vitro and in vivo. Oncol Rep 35: 43-49, 2016.

16. Kim EL, Wüstenberg R, Rübsam A, Schmitz-Salue C, Warnecke G, Bücker EM, Pettkus N, Speidel D, Rohde V, Schulz-Schaeffer W, et al: Chloroquine activates the $\mathrm{p} 53$ pathway and induces apoptosis in human glioma cells. Neruo-Oncol 12: 389-400, 2010

17. Lakhter AJ, Sahu RP, Sun Y, Kaufmann WK, Androphy EJ, Travers JB and Naidu SR: Chloroquine promotes apoptosis in melanoma cells by inhibiting BH3 domain-mediated PUMA degradation. J Invest Dermatol 133: 2247-2254, 2013.

18. Mizushima N, Yoshimori T and Levine B: Methods in mammalian autophagy research. Cell 140: 313-326, 2010.

19. Maiuri MC, Zalckvar E, Kimchi A and Kroemer G: Self-eating and self-killing: Crosstalk between autophagy and apoptosis. Nat Rev Mol Cell Biol 8: 741-752, 2007.

20. Kroemer G and Levine B: Autophagic cell death: The story of a misnomer. Nat Rev Mol Cell Biol 9: 1004-1010, 2008.

21. Kaminskyy V, Abdi A and Zhivotovsky B: A quantitative assay for the monitoring of autophagosome accumulation in different phases of the cell cycle. Autophagy 7: 83-90, 2011

22. Yan Y, Jiang K, Liu P, Zhang X, Dong X, Gao J, Liu Q, Barr MP, Zhang Q, Hou X, Meng S and Gong P: Bafilomycin A1 induces caspase-independent cell death in hepatocellular carcinoma cells via targeting of autophagy and MAPK pathways. Sci Rep 6: $37052,2016$.

23. Wang L, Gao C, Yao S and Xie S: Blocking autophagic flux enhances matrine-induced apoptosis in human hepatoma cells. Int J Mol Sci 14: 23212-23230, 2013.

24. Sheppard KE, Kinross KM, Solomon B, Pearson RB and Phillips WA: Targeting PI3 kinase/Akt/mTOR signaling in cancer. Crit Rev Oncog 17: 69-95, 2012.

25. Liu K, Liu P, Liu R and Wu X: Dual AO/EB staining to detect apoptosis in osteosarcoma cells compared with flow cytometry. Med Sci Monit Basic Res 21: 15-20, 2015.

26. Zhao X, Ma S, Liu N, Liu J and Wang W: A polysaccharide from Trametes robiniophila Murrill induces apoptosis through intrinsic mitochondrial pathway in human osteosarcoma (U-2 OS) cells. Tumor Biol 36: 5255-5263, 2015. 
27. Thomé R, Lopes SC, Costa FT and Verinaud L: Chloroquine: Modes of action of an undervalued drug. Immunol Lett 153: $50-57,2013$.

28. Zhao XG, Sun RJ, Yang XY, Liu DY, Lei DP, Jin T and Pan XL: Chloroquine-enhanced efficacy of cisplatin in the treatment of hypopharyngeal carcinoma in xenograft mice. PLoS One 10 e0126147, 2015.

29. Yue L, Mei Q, Zhe W, Zhang W, Liu D, and Liu Y: Study of chloroquine on anti-tumor effects of $\mathrm{S}_{180}$ tumor-bearing mice and its mechanisms. Acta Anatomica Sinica 6: 779-784, 2016.

30. Mizushima N: Methods for monitoring autophagy. Int J Biochem Cell Biol 36: 2491-2502, 2004.

31. Kang R, Zeh HJ, Lotze MT and Tang D: The Beclin 1 network regulates autophagy and apoptosis. Cell Death Differ 18: 571-580, 2011.

32. Maiuri MC, Criollo A and Kroemer G: Crosstalk between apoptosis and autophagy within the Beclin 1 interactome. EMBO J 29: 515-516, 2010.
33. Heras-Sandoval D, Pérez-Rojas JM, Hernández-Damián J and Pedraza-Chaverri J: The role of PI3K/AKT/mTOR pathway in the modulation of autophagy and the clearance of protein aggregates in neurodegeneration. Cell Signal 26: 2694-2701, 2014.

34. Datta K, Suman S and Fornace AJ Jr, Radiation persistently promoted oxidative stress, activated mTOR via PI3K/Akt, and downregulated autophagy pathway in mouse intestine. Int J Biochem Cell Biol 57: 167-176, 2014.

35. Mariño G, Niso-Santano M, Baehrecke EH and Kroemer G: Self-consumption: The interplay of autophagy and apoptosis. Nat Rev Mol Cell Biol 15: 81-94, 2014.

36. Yue $\mathrm{Z}$ and Zhong Y: From a global view to focused examination: Understanding cellular function of lipid kinase VPS34-Beclin 1 complex in autophagy. J Mol Cell Biol 2: 305-307, 2010.

37. Ciechomska IA, Goemans GC, Skepper JN and Tolkovsky AM: Bcl-2 complexed with Beclin-1 maintains full anti-apoptotic function. Oncogene 28: 2128-2141, 2009. 